\title{
Oncolytic Seneca Valley Virus: past perspectives and future directions
}

This article was published in the following Dove Press journal:

Oncolytic Virotherapy

6 September 2016

Number of times this article has been viewed

\author{
Michael J Burke \\ Department of Pediatrics, Division of \\ Pediatric Oncology, Medical College \\ of Wisconsin, MACC Fund Research \\ Center, Milwaukee, WI, USA
}

\begin{abstract}
Seneca Valley Virus isolate 001 (SVV-001) is an oncolytic RNA virus of the Picornaviridae family. It is also the first picornavirus discovered of the novel genus Senecavirus. SVV-001 replicates through an RNA intermediate, bypassing a DNA phase, and is unable to integrate into the host genome. SVV-001 was originally discovered as a contaminant in the cell culture of fetal retinoblasts and has since been identified as a potent oncolytic virus against tumors of neuroendocrine origin. SVV-001 has a number of features that make it an attractive oncolytic virus, namely, its ability to target and penetrate solid tumors via intravenous administration, inability for insertional mutagenesis, and being a self-replicating RNA virus with selective tropism for cancer cells. SVV-001 has been studied in both pediatric and adult early phase studies reporting safety and some clinical efficacy, albeit primarily in adult tumors. This review summarizes the current knowledge of SVV-001 and what its future as an oncolytic virus may hold.
\end{abstract}

Keywords: oncolytic, virus, oncology, Seneca, valley

\section{Introduction}

Seneca Valley Virus isolate 001 (SVV-001) is an oncolytic RNA virus belonging to the Picornaviridae family that replicates through an RNA intermediate and lacks the ability to integrate into the host genome. SVV-001 does not have any reverse transcriptase activity and does not go through a DNA phase during replication. SVV-001 was originally discovered by Genetic Therapy Inc. (Gaithersburg, MD, USA) in 2002 as a contaminant in cell culture of human fetal retinoblasts, believed to be introduced through the bovine serum or porcine trypsin that was in the culture media. ${ }^{1}$ When SVV-001 was first isolated, purified, and analyzed, it had a similar profile to the cardioviruses and was thought initially to have arisen as a recombinant virus derived from a cardiovirus. ${ }^{1-3}$ But after further in-depth analysis, including electron microscopy and complete nucleotide sequencing, where the genomic sequence of SVV-001 was compared with that of other cardioviruses, this new virion was found to be most consistent with the family Picornaviridae and not a recombinant derived from any known cardiovirus. ${ }^{1}$ Therefore, based on the similar viral features and replication patterns, this new discovery was placed into the picornavirus family and given its own designation as SVV-001.

SVV-001 is homologous to and serologically related to 12 viruses isolated from pig specimens in the US, and it is nonpathogenic in both human and animal species. ${ }^{4}$ Viruses closely related to SVV-001 ( $>95 \%$ sequence identity) have been isolated by 
the United States Department of Agriculture (USDA) from pigs on at least 12 occasions in California, Illinois, Iowa, Louisiana, Minnesota, New Jersey, and North Carolina. ${ }^{1}$ These viruses are also serologically linked as antisera that were raised against one of these viruses that cross-reacted with all the isolates, including SVV-001. Analysis of animal sera demonstrates that neutralizing antibodies to SVV-001 are present in 27 out of 71 porcine samples, ten out of 30 bovine samples, and five out of 35 wild mouse samples. No neutralizing antibodies were found in 52 nonhuman primate sera representing four species. Among $>100$ human serum samples analyzed to date, one was found to have neutralizing antibodies to SVV-001 (titer or dilution of sera that neutralized $100 \%$ of the virus: $1: 8) .{ }^{4}$ Taken together, these data suggest that SVV-001 and/or closely related viruses may naturally replicate in farm animals, but previous exposure to SVV-001 is relatively rare in human beings. To date, there is no evidence that SVV-001 or the related viruses cause harmful disease in farm animals. The transmissibility of SVV-001 appears not to be by either aerosol or contact as experiments with mice injected with SVV-001 cohoused with naïve adult mice did not result in the development of neutralizing antibodies in any of the naïve mice. ${ }^{5}$

The $5^{\prime}$ untranslated region of the picornavirus genome contains an internal ribosome entry site (IRES) that directs cap-independent internal initiation of protein synthesis with four distinct classes previously described. ${ }^{6-8}$ Interestingly, the IRES found in SVV-001 more closely resembles the IRES found in hepatitis $\mathrm{C}$ virus within the Flaviviridae family and classical swine fever virus, a pestivirus also a member of Flaviviridae, rather than the IRES elements of Picornaviridae. ${ }^{9}$ A sequence alignment of IRES elements between SVV-001 and hepatitis $\mathrm{C}$ virus and classical swine fever virus identified $52 \%$ and $47 \%$ identity similarity between SVV-001 and hepatitis C virus and SVV-001 and classical swine fever virus, respectively. ${ }^{9}$ These similarities appeared to only occur in the domain regions of IIIa, IIIe, and IIIc and the short motifs within domains II and IIId1. ${ }^{9}$ Based on the findings of similar IRES elements across the two viral families in Picornaviridae and Flaviviridae, the possibility of genetic exchange between several different members of these virus families is suggested. ${ }^{9}$

Picornaviruses that are relevant to human disease include rhinoviruses (common cold), cardioviruses (myocarditis), hepatoviruses (hepatitis), and enteroviruses (diarrhea and meningitis). To date, there is no evidence that SVV-001 causes any harmful disease in any species, and exposure to SVV-001 does not appear to be prevalent in the human population. ${ }^{4} \mathrm{SVV}-001$ is a self-replicating virus that rapidly enters tumor cells and has been shown to induce cytotoxicity in tumors expressing neuroendocrine features, such as synaptophysin, chromogranin A, and neuron-specific enolase, in several in vitro and in vivo models. ${ }^{10,11}$ Tumors expressing such neuroendocrine features and shown to be susceptible to SVV-001 killing include neuroblastoma, rhabdomyosarcoma, medulloblastoma, Wilms tumor, glioblastoma, as well as adult small-cell lung cancer. ${ }^{4,10,12}$ Thus, SVV-001 appeared to be an ideal oncolytic virus given its ability to penetrate tumors and rapidly replicate, having a nonintegrating RNA genome, being nonpathogenic, having a low incidence of preexisting immunity in humans, and being selective to tumors expressing neuroendocrine features (Figure 1). This review summarizes both the preclinical and clinical data surrounding SVV-001, including results from the first-in-human and first-in-children Phase I clinical trials, concluding with the author's perspectives as to what the future may hold for SVV-001 as a viable option in viral oncotherapy.

\section{In vitro activity of SVV-00 I}

The structure of SVV-001 includes four subunits, VP1, VP2, VP3, and VP4, that have a similar fold pattern as the corresponding proteins of other Picornaviridae viruses. ${ }^{2}$ Because SVV-001 has the ability to target and kill cells with neuroendocrine features, it is possible that this cell tropism is guided by the binding of receptors expressed on these tumor cells. However, to date, this has not been validated using the structure of SVV-001. What has been identified based on the SVV-001 structure, which may result in neuroendocrine protein binding, includes a variety of motifs on the surface or near the surface in depressions or canyons of SVV-001 that may bind to specific integrins that are present on tumor cells (eg, $\alpha_{1} \beta_{4}$ on small-cell lung carcinoma [SCLC] $)^{2}$ Liu et al identified $\alpha 2,3$ - and $\alpha 2,6$-linked sialic acids as necessary for SVV-001 infection in pediatric glioblastoma multiforme (GBM) cell lines where blocking of these proteins resulted in synergistic inhibition of SVV-001 infection. Other proteins that SVV-001 may have the ability to target and bind include low-density lipoprotein receptors that are recognized by other viruses, including members of the rhinovirus. Whether SVV-001 is able to bind directly to these proteins or only after local conformational changes is made to expose the motif and aid in receptor binding being unclear.

SVV-001, like other members of the Picornaviridae family, kills cells through intracellular viral replication resulting in cell lysis and autophagy and appears to be selective toward tumors that express neuroendocrine properties. As demonstrated by Reddy et al, ${ }^{4} 13$ out of 23 small-cell lung cancer cell lines tested were sensitive to SVV-001-mediated killing 


\section{Picornavirus}

Small virus able to penetrate tumor mass

Rapid replication and cell lysis

Nonintegrating RNA genome

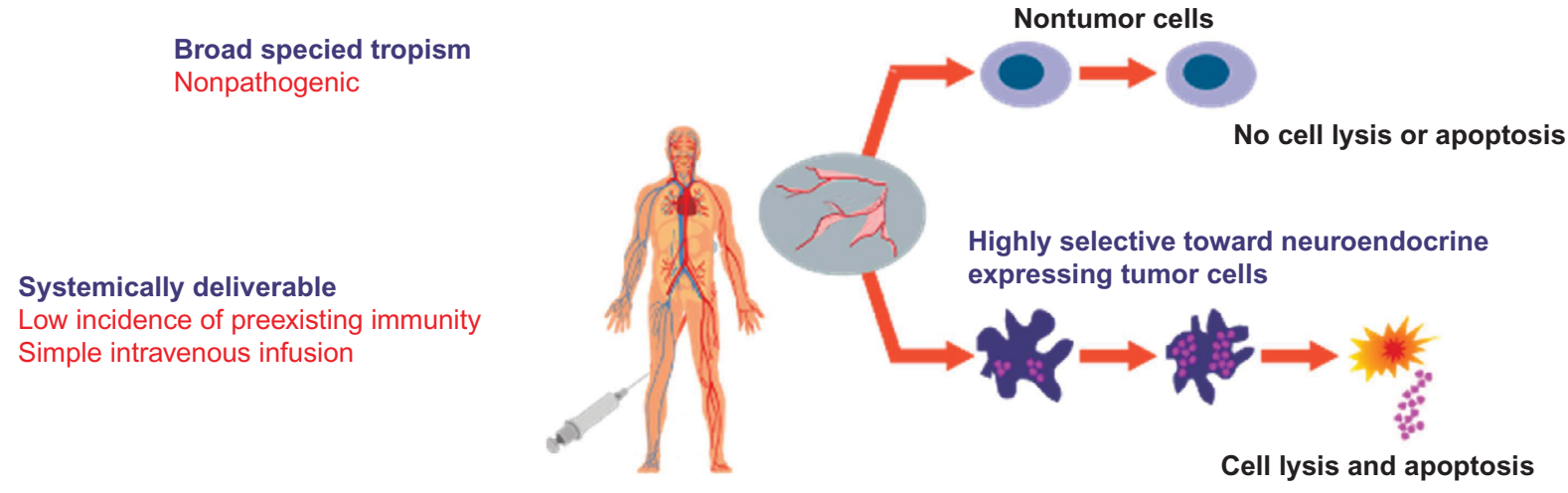

Genetically stable

Nonengineered native virus has no revertant from

Manufacturing

Grows to high titer in an

FDA-approved cell line

Figure I Properties of SVV-00I as an oncolytic virus.

Notes: The cartoon demonstrates SVV-00I being administered into a patient with a neuroendocrine tumor as an IV infusion. SVV-00I displays tropism to the tumor resulting in intratumoral replication, cell lysis, and cell death but does not replicate within or destroy nontumor cells, showing its selectivity as an oncolytic virus.

Abbreviations: SVV-00I, Seneca Valley Virus isolate 00I; IV, intravenous; FDA, US Food and Drug Administration.

with half maximal effective concentration $\left(\mathrm{EC}_{50}\right)$ values $<10$. As well, the authors identified seven out of eight pediatric neuroendocrine cancer cell lines and two out of three adrenal gland cortical carcinoma cell lines that were permissive to SVV-001 and resulted in cell death. SVV-001 was also tested against noncancer human cells, primary hepatocytes, and cancer cell lines not expressing neuroendocrine properties, which were all resistant to SVV-001-mediated cell killing.

The Pediatric Preclinical Testing Program (PPTP), operated by St. Jude Children's Research Hospital and sponsored by the National Cancer Institute (NCI), evaluated SVV-001 against 23 cancer cell lines using 96-hour exposures and identified cell killing, $\mathrm{IC}_{50}$ (the agent concentration to kill $50 \%$ of untreated cells) down to less than one virus particle per cell, in three out of four neuroblastoma, two out of four rhabdomyosarcoma, and one out of four Ewing sarcoma cell lines. ${ }^{10}$ The results of the abovementioned in vitro studies, demonstrating cell killing at very low viral concentrations, identified SVV-001 as a potent oncolytic virus toward neuroendocrine cancer cell lines and a potential therapy for recurrent/resistant tumors expressing neuroendocrine features.

\section{In vivo activity of SVV-00 I}

SVV-001 has been studied in a number of in vivo models. Using an orthotopic xenograft Rag2 severe combined immunodeficiency (SCID) mouse model of pediatric GBM, Liu et $\mathrm{l}^{13}$ injected SVV-001 $\left(5 \times 10^{12} \mathrm{vp} / \mathrm{kg}\right)$ into the tail vein of ten immunodeficient mice at 2 weeks or 4 weeks after tumor injection and compared to controls $(n=10)$ injected with saline. Mice were harvested once neurological deficits developed. In a separate group, xenograft tumor cells were allowed to grow for $\sim 8$ weeks prior to SVV-001 injection, after which brains were harvested on days $1,2,4$, or 7 postSVV-001 injection. SVV-001-infected glial cells were identified as early as 24 hours post-viral injection with evidence of cell lysis by 4 days. SVV-001 significantly prolonged survival compared to controls $(P<0.001)$ and completely eliminated xenograft tumors in two $(20 \%)$ and eight $(80 \%)$ of the ten mice treated at 2 weeks or 4 weeks, respectively, after tumor injection. Importantly, SVV-001 did not infect any adjacent nontumor brain cells or normal glial cells in the cerebral gray or white matter.

In a similar study using Rag2 SCID mice, Yu et al developed a comparable orthotopic xenograft to their GBM mouse model but instead injected medulloblastoma cells. ${ }^{11}$ Then taking 20 of these immunodeficient mice, SVV-001 $\left(5 \times 10^{12} \mathrm{vp} / \mathrm{kg}\right)$ was injected at 2 weeks $(\mathrm{n}=10)$ or 4 weeks $(n=10)$ posttumor injection and compared to ten control mice injected with saline. The mice that developed neurological deficits were harvested for histopathological analysis. In a 
separate group, xenograft tumor cells were allowed to grow for $\sim 8$ weeks prior to SVV-001 injection, after which brains were harvested on days 1,2 , or 6 post-SVV-001 injection. The results reported that $>75 \%$ of tumor cells in the core area and $\sim 100 \%$ of metastatic tumor cells were infected with SVV-001 at 48 hours with diffuse viral penetration into both tumor and metastatic sites at day 6. Mice receiving SVV-001 had significantly greater survival compared to controls with a median of $141.6 \pm 25.2$ days in mice with smaller tumors receiving SVV-001 compared to their matched controls (63.8 \pm 4.2 days; $P<0.00001)$ and even longer survival than mice with larger tumors $(187.9 \pm 20.3$ days; $P<0.001)$. Even more impressive was that eight of ten long-term survivors had complete elimination of their tumors after a single injection of SVV-001.

SVV-001 was further studied in SCID mice injected with Y79 retinoblastoma $\left(1 \times 10^{6}\right.$ cells $)$ into the flank. ${ }^{4}$ Complete Y79 retinoblastoma tumor regression was observed in six out of eight $(75 \%)$ mice injected with a single dose of SVV-001 at $1 \times 10^{8} \mathrm{vp} / \mathrm{kg}$, seven out of seven mice $(100 \%)$ injected at $1 \times 10^{11} \mathrm{vp} / \mathrm{kg}$, and five out of seven mice $(71 \%)$ treated at the highest dose of $1 \times 10^{14} \mathrm{vp} / \mathrm{kg}$ with all mice with eradicated tumors surviving to study end (day 84) without evidence of tumor recurrence. In another human xenograft retinoblastoma (Y79 cells) model investigating the oncolytic effect of SVV-001, 20 Rag2 SCID mice were injected with SVV-001 $\left(1 \times 10^{13} \mathrm{vp} / \mathrm{kg}\right)$ into the tail vein 3 weeks after $Y 79$ intraocular injection and sacrificed 2 weeks later and compared to 20 controls. ${ }^{14}$ Of the treated mice, only one had a grossly visible tumor at the time of harvest. The remaining mice had small localized tumors that could only be observed histopathologically and had preservation of both lens and globe. In marked contrast to the untreated control mice, all mice receiving SVV-001 had normal activity and appetite and were not in any visible discomfort. ${ }^{14}$

SVV-001 was tested against an in vivo panel of xenograft models in the Pediatric Preclinical Testing Program. ${ }^{10} \mathrm{~A}$ single dose of SVV-001 at $3 \times 10^{12} \mathrm{vp} / \mathrm{kg}$ was injected into 357 mice with a variety of tumors, including Ewing sarcoma, osteosarcoma, medulloblastoma, rhabdomyosarcoma, and neuroblastoma. Objective responses including complete response and maintained complete response were observed in four out of four mice with alveolar rhabdomyosarcoma and four out of five mice with neuroblastoma (Figure 2). ${ }^{10}$ There were no objective responses in the mice with Ewing sarcoma, osteosarcoma, or medulloblastoma.

Similar to the results observed in the in vitro studies of SVV-001, the murine in vivo investigations described earlier report dramatic results after only single injections of SVV-001 into mice harboring rhabdomyosarcoma, neuroblastoma, GBM, medulloblastoma, or retinoblastoma tumors, including complete eradication of tumor in some cases. Based on the very encouraging preclinical results and the favorable safety profile observed from the animal studies, ${ }^{4,11,13}$ particularly with SVV-001 not infecting nontumor cells, an adult Phase I study was planned for patients with neuroendocrine-expressing tumors.

\section{Clinical trials of SVV-00 I}

SVV-001 has been successfully tested in human Phase I clinical trials of both adults and children demonstrating the safety and feasibility of infusing this oncolytic virus in the patients with malignancies. The adult trial was a first-in-human and first-in-class (picornavirus) trial for SVV-001 and set the stage for this therapy to be tested in children which followed shortly after through the Children's Oncology Group (COG).

\section{Adult trials}

The adult Phase I trial of SVV-001 (NCT00314925) sought to evaluate the safety, viral kinetics, and viral dynamics in patients with advanced solid tumors demonstrating expression of neuroendocrine markers defined as immunohistochemical expression of CD56, chromogranin A, and/or synaptophysin. ${ }^{15}$ The patients were to have advanced disease for which there were no standard therapies of proven benefit available. The study was a dose escalation of SVV-001 over five dose levels ranging from $10^{7}$ to $10^{11} \mathrm{vp} / \mathrm{kg}$ as a single intravenous (IV) dose. The primary study objectives were toxicity assessment and to determine a recommended Phase II dose. The secondary objectives included measuring viral titers in the blood, sputum, urine, stool, and nasal swabs, as well as titers of neutralizing antibodies at each dose level. NCI Common Terminology Criteria for Adverse Events Version 3.0 was used for toxicity attribution, and antitumor response was assessed by serial computed tomography scans using Response Evaluation Criteria in Solid Tumors (RECIST) Version 1.0 (National Cancer Institute, Bethesda, MD, USA).

Thirty patients with a median age of 60 years (range 32-73 years) were treated in the study. The diagnoses included six patients with SCLC and 24 with mixed neuroendocrine tumors of which six had carcinoid tumors. There were no dose-limiting toxicities (DLTs) reported at any dose level and no maximum tolerated dose defined. The most common toxicities reported were grade 1 fever ( $33 \%$, all dose levels) and grade 1 fatigue (42\%, dose levels $2-4$ and $17 \%$, dose level 5). There was a single grade 3 adverse event reported 


\begin{tabular}{|c|c|c|c|}
\hline Xenograft line & Histology & $\begin{array}{c}\text { Overall group } \\
\text { response }\end{array}$ & Heat map \\
\hline Rh10 & $\begin{array}{c}\text { Alveolar } \\
\text { rhabdomyosarcoma }\end{array}$ & $\mathrm{CR}$ & \\
\hline Rh28 & $\begin{array}{c}\text { Alveolar } \\
\text { rhabdomyosarcoma }\end{array}$ & MCR & \\
\hline Rh30 & $\begin{array}{c}\text { Alveolar } \\
\text { rhabdomyosarcoma }\end{array}$ & CR & \\
\hline Rh30R & $\begin{array}{c}\text { Alveolar } \\
\text { rhabdomyosarcoma }\end{array}$ & MCR & \\
\hline NB-SD & Neuroblastoma & CR & \\
\hline NB-1771 & Neuroblastoma & MCR & \\
\hline NB-1691 & Neuroblastoma & MCR & \\
\hline NB-EBc1 & Neuroblastoma & PD & \\
\hline NB-1643 & Neuroblastoma & MCR & \\
\hline EW5 & Ewing sarcoma & PD & \\
\hline EW8 & Ewing sarcoma & PD & \\
\hline OS-1 & Osteosarcoma & PD & \\
\hline OS-2 & Osteosarcoma & PD & \\
\hline BT-28 & Medulloblastoma & PD & \\
\hline BT-45 & Medulloblastoma & PD & \\
\hline BT-50 & Medulloblastoma & PD & \\
\hline
\end{tabular}

Figure 2 SVV-00I in vivo response.

Notes: The heat map represents tumor response in PPTP xenograft models after a single injection of SVV-00I $\left(3^{\prime} 10^{12} \mathrm{vP} / \mathrm{kg}\right)$. A high level of antitumor activity is shown in red (MCR) and orange (CR) with no activity in green (PD). Adapted with permission from John Wiley and Sons. Morton CL, Houghton PJ, Kolb EA, et al. Initial testing of the replication competent Seneca Valley virus (NTX-0I0) by the pediatric preclinical testing program. Pediatr Blood Cancer. 2010;55(2):295-303. ${ }^{10}$ (C) 2010 John Wiley and Sons. Abbreviations: SVV-00I, Seneca Valley Virus isolate 00I; PPTP, Pediatric Preclinical Testing Program; MCR, maintained complete response; CR, complete response; PD, progressive disease.

at dose level 5 with lymphopenia. An additional 12 patients were treated at dose level $5\left(10^{11} \mathrm{vp} / \mathrm{kg}\right)$ to further assess for toxicity at the highest dose level, and no DLT was reported.

Although no patient demonstrated an objective response based on RECIST criteria for complete or partial response, one patient with SCLC reported stable disease persisting for 10 months post-SVV-001 and remained alive 3 years after SVV-001 treatment and at the time of last study contact. Five additional patients with non-small-cell carcinoma tumors experienced some minor tumor reductions although not meeting RECIST for partial response. SVV-001 viral serum titers in the majority of subjects were highest on the day of viral administration with the day 1 peak increasing by cohort, reflecting the log increments in the administered dose. ${ }^{15}$ Neutralizing antibodies developed in all patients within 2 weeks of receiving SVV-001, and all patients cleared virus from all compartments (blood, urine, stool, sputum, and nasal secretions) concurrent with or following the neutralizing antibody response.

In a patient with SCLC with extensive liver metastases who died on study of progressive disease after SVV-001 administration, an autopsy was performed to see if SVV-001 was present in tumor and nontumor tissues. Immunohistochemical analyses demonstrated no detectable SVV-001 particles in nontumor tissues, which included kidney and pancreas, but in contrast, the metastatic liver lesions demonstrated intense intracellular staining for viral particles in the tumor cells but without evidence of virus in the adjacent normal liver. Thus, it appeared that IV administration of SVV001 was associated with highly specific intratumoral infection and replication of SVV-001 without evidence of infecting or replicating in the normal, nontumor cells. Of further interest 
is that this patient did have the presence of neutralizing antibodies, and the autopsy performed 4 weeks after SVV-001 was infused suggesting persistent viral replication.

Overall, this first-in-human Phase I study of SVV-001 proved safety and feasibility of IV delivery to adult patients with heavily pretreated and metastatic neuroendocrine tumors. All patients cleared virus within the study period and mounted immunity with neutralizing antibodies. The identified and recommended Phase II dose from this study was $1 \times 10^{11} \mathrm{vp} / \mathrm{kg}$. Based on the results from this trial, a randomized double-blinded Phase II study of SVV-001 in patients with extensive-stage small-cell lung cancer is ongoing but not currently recruiting patients (NCT01017601). The patients of this study are randomized to a single dose of SVV-001 $\left(1 \times 10^{11} \mathrm{vp} / \mathrm{kg}\right)$ on day 1 versus a placebo infusion. As well, there are no other active studies investigating SVV-001 in adult patients registered with clinicaltrials.gov.

\section{Pediatric trials}

Based on the safety results of the adult Phase I SVV-001 study and the strong preclinical data where SVV-001 induced cytotoxicity in several in vitro and in vivo models of pediatric tumors, including neuroblastoma, rhabdomyosarcoma and medulloblastoma, ${ }^{10,11}$ a pediatric Phase I study was developed (NCT01048892) in children with neuroendocrine tumors. ${ }^{16}$ Patients of age 3-21 years with refractory tumors and histologic confirmation of neuroblastoma, rhabdomyosarcoma, Wilms tumor, retinoblastoma, carcinoid tumor, or adrenal cortical carcinoma were eligible. Similar to the adult trial, SVV-001 was administered as a single IV infusion and patients were followed for at least 28 days or until viral clearance,

A

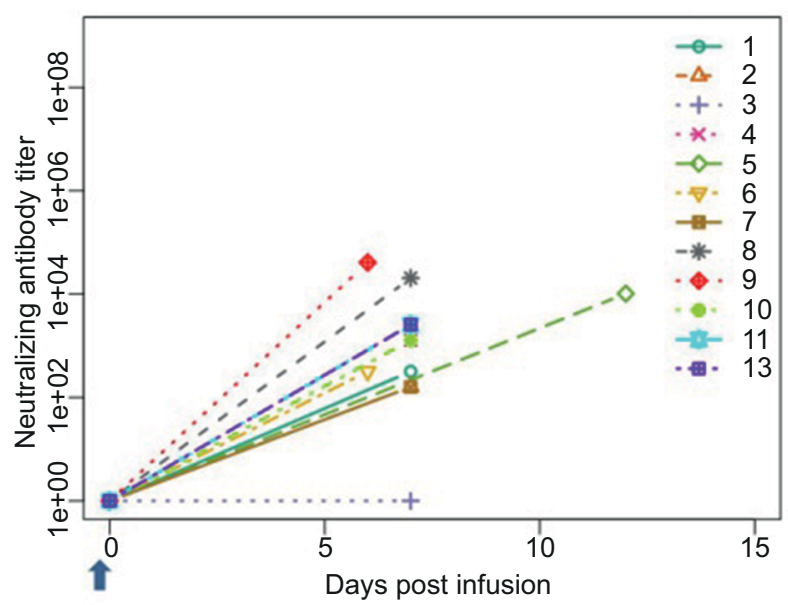

whichever was longer. Three dose levels were investigated in Part A ( $\left.10^{9} \mathrm{vp} / \mathrm{kg}-10^{11} \mathrm{vp} / \mathrm{kg}\right)$, and low-dose metronomic and IV cyclophosphamide was added to dose level $3\left(10^{11} \mathrm{vp} /\right.$ $\mathrm{kg}$ ) in Part B as immunomodulatory therapy. As well in Part B, two infusions of SVV-001 were administered, one on day 8 and another on day 29 , in an attempt to improve antitumor activity through multiple infusions. The primary study objectives were to estimate the maximum tolerated dose and/or the recommended Phase II dose of SVV-001 as a single infusion and in combination with low-dose cyclophosphamide with two consecutive infusions. The secondary objectives included defining antitumor activity of SVV-001 and development of neutralizing antibodies following a single infusion and the combination of SVV-001 and cyclophosphamide. NCI Common Terminology Criteria for Adverse Events Version 4.0 (National Cancer Institute, Bethesda, MD, USA) was used for toxicity attribution, and antitumor response was assessed using a modified RECIST.

Twenty-two patients enrolled with a median age of 8.8 years (range, 4.8-18.3 years). Four patients were not evaluable for DLT assessment leaving 18 evaluable, 12 treated on Part A and six on Part B. The diagnoses included neuroblastoma $(n=12)$, adrenal cortical carcinoma $(n=3)$, Wilms tumor $(n=3)$, rhabdomyosarcoma $(n=3)$, and carcinoid tumor $(n=1)$. A single DLT was reported in Part A (grade 3 pain) at dose level $1\left(10^{9} \mathrm{vp} /\right.$ $\mathrm{kg}$ ) with no further DLTs reported at any other dose level in Part A or B. There were no objective responses (complete or partial) reported for Part A or B; however, six patients $(50 \%)$ in Part A and four patients (67.7\%) in Part B maintained stable disease at the time of disease evaluation. Patients in Part A and B cleared SVV-001 from both compartments tested (blood

B

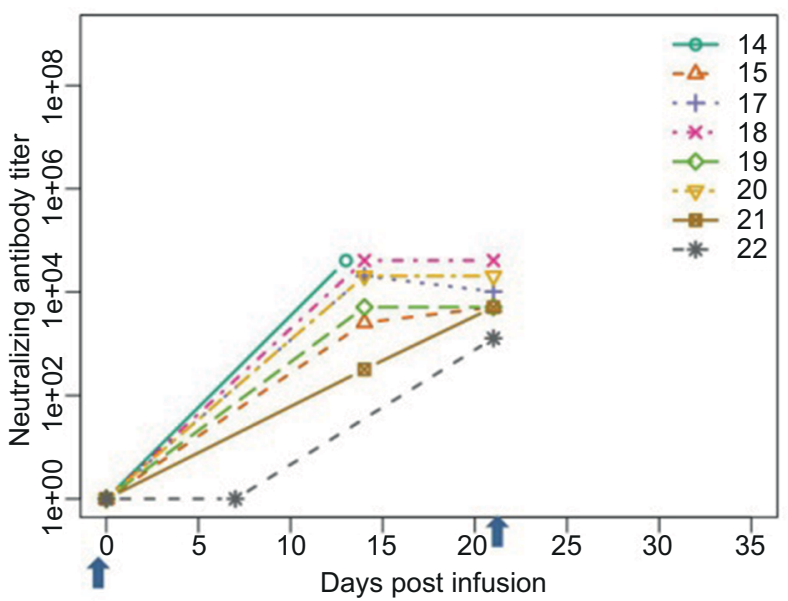

Figure 3 Neutralizing antibodies to SVV-00I.

Notes: Development of neutralizing antibodies over time after a single dose (A) and after two doses (B) of SVV-00I in the COG ADVL09II pediatric trial. The arrow denotes timing of SVV-00I administration. Reproduced with permission from John Wiley and Sons. Burke MJ, Ahern C, Weigel BJ, et al. Phase I trial of Seneca Valley virus (NTX-0I0) in children with relapsed/refractory solid tumors: a report of the Children's Oncology Group. Pediatr Blood Cancer. 20I5;62(5):743-750.'16 @ 20I5 John Wiley and Sons. Abbreviations: SVV-00I, Seneca Valley Virus isolate 00I; COG, Children's Oncology Group. 
A
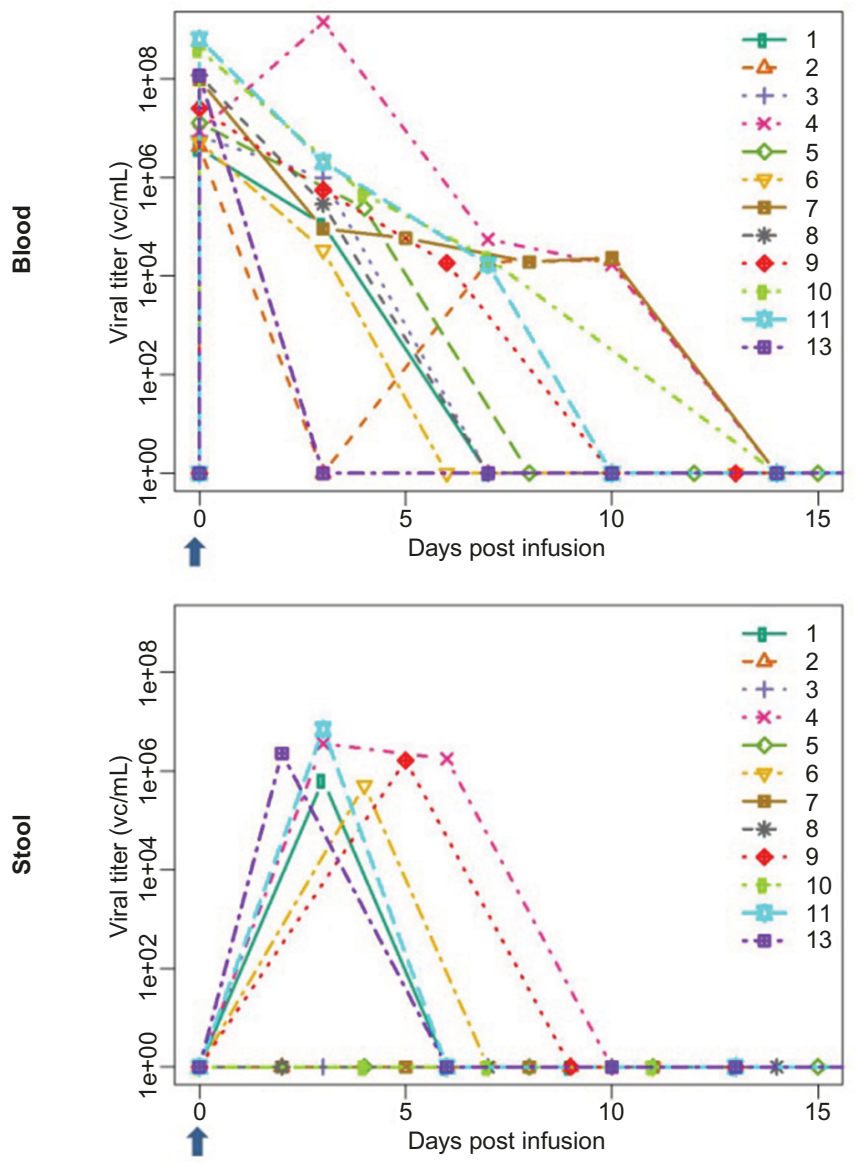

Part B

B
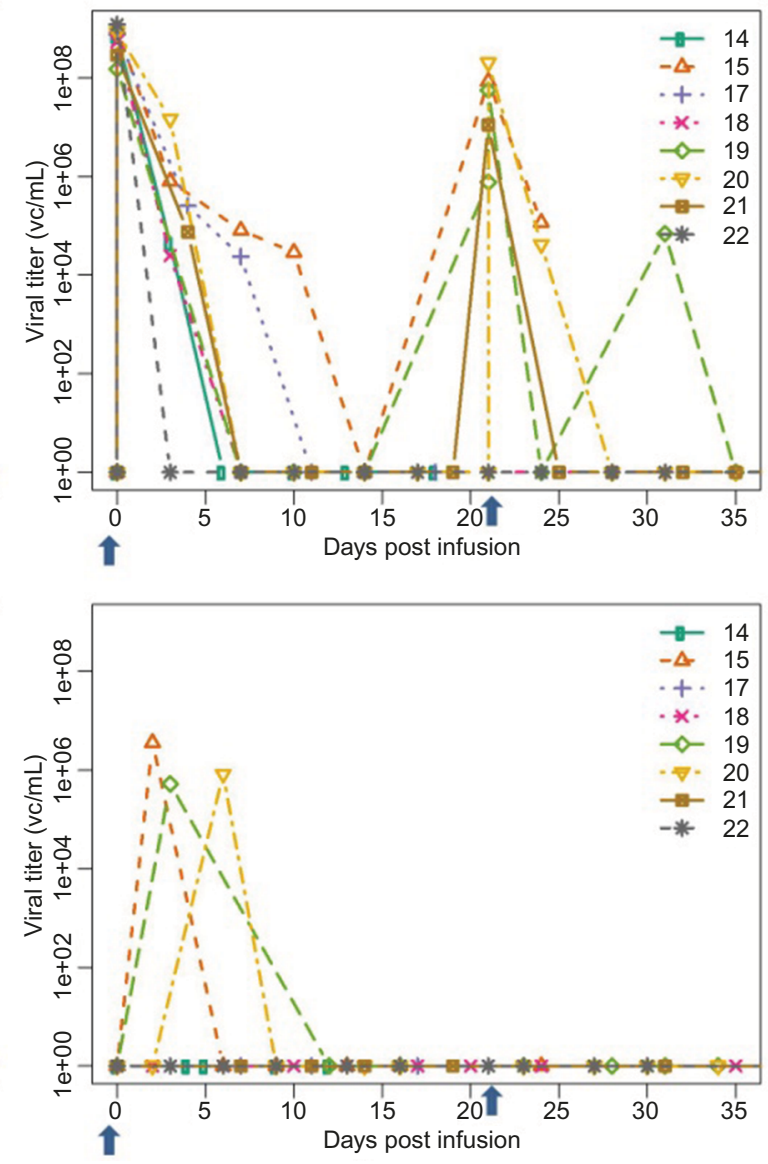

Figure 4 Viral clearance of SVV-00I.

Notes: Viral clearance in the blood and stool over time after a single dose (A) and after two doses (B) of SVV-00I in the COG ADVL09II pediatric trial. The arrow denotes timing of SVV-00I administration. Reproduced with permission from John Wiley and Sons. Burke MJ, Ahern C, Weigel BJ, et al. Phase I trial of Seneca Valley virus (NTX0I0) in children with relapsed/refractory solid tumors: a report of the Children's Oncology Group. Pediatr Blood Cancer. 2015;62(5):743-750. ${ }^{16}$ (C) 20I5 John Wiley and Sons. Abbreviations: SVV-00I, Seneca Valley Virus isolate 00I; COG, Children's Oncology Group.

and stool) within 3 weeks of their viral infusion (Figure 3), and neutralizing antibodies were identified in eleven out of 12 patients in Part A and all patients in Part B despite the addition of cyclophosphamide (Figure 4).

This study was the first Phase I clinical trial of children investigated on an oncolytic virus, SVV-001, demonstrating tolerability both as a single IV infusion as well as in combination with low-dose cyclophosphamide. Despite the addition of immunomodulatory therapy in cyclophosphamide in Part B, all patients cleared SVV-001 from their blood and stool, developed neutralizing antibodies, and did not report an objective response. Currently, there is no COG pediatric Phase II study of SVV-001 or any plans for development. As well, there are no other active studies investigating SVV-001 in pediatric patients registered with clinicaltrials.gov.

\section{Conclusion}

SVV-001 is the first oncolytic virus to be tested in both a pediatric and adult Phase I trial for recurrent/refractory tumors and the first oncolytic virus ever to be tested in children worldwide. Despite encouraging preclinical data in SCID mice supporting the use of this virus in tumors expressing neuroendocrine features, objective clinical responses were lacking in both the adult and pediatric Phase I studies. This is somewhat surprising given just how potent this virus appeared in the SCID mouse models with complete tumor eradication after a single infusion of SVV-001. This may have been in part due to the severe immunocompromised state of these mice, being Rag2 SCID models, which lacked the ability to mount a T-regulatory cell immune response and/or develop neutralizing antibodies toward SVV-001, as was seen in the human studies. However, systemic administration of the virus did prove to be safe and feasible in both children and adults without identification of a maximum tolerated dose.

The pediatric COG trial was the first to attempt immunomodulation of SVV-001 to delay development of neutralizing antibodies and viral clearance using low-dose metronomic 
and IV cyclophosphamide. This strategy had previously been shown to enhance the oncolytic activity of an adenovirus in adult patients with metastatic tumors who progressed on conventional therapy. ${ }^{17}$ In this study, patients who received oncolytic adenovirus plus metronomic and IV cyclophosphamide had significantly lower numbers of T-regulatory cells posttreatment $(P=0.032)$ and greater disease control ( $77 \%$ versus $22 \%)$ compared to those who received oncolytic adenovirus alone. Despite the attempt to delay viral clearance and antibody formation in the COG study with oral and IV cyclophosphamide, all patients cleared virus and rapidly developed neutralizing antibodies. It appears, at least in the case of SVV-001, that lowdose metronomic and IV cyclophosphamide is not enough to blunt the immune system in patients or enhance the antitumor effect. It appears that even in heavily pretreated and severely immunocompromised patients, there is enough of an intact immune response to not only identify viral immunogenicity but also mount an antiviral response strong enough to eliminate SVV-001 and develop antibodies upon initial and repeat exposure. Thus, any future studies of SVV-001 will need to focus on strategies to minimize the development of neutralizing antibodies, which are likely negatively affecting treatment response in both children and adults with neuroendocrine tumors. One potential strategy would be in combining SVV001 with a peptide prodrug linked to a cytotoxic agent that is activated by the viral protease once inside the tumor cell, resulting in not only tumor cell death but also cytotoxicity to adjacent uninfected cells via the bystander effect. ${ }^{18}$

Potential biomarkers predicting clinical response to the oncolytic activity of SVV-001 was investigated in the COG Phase I trial using diagnostic tumor tissue. ${ }^{16}$ The available tumor samples were evaluated for the expression of genes induced by the type I interferon response to correlate with intratumoral replication and viral persistence. The only gene identified that may be predictive of SVV-001 infection was TLR3, which detects the pathogen-associated molecular pattern of dsRNA used as a replicative intermediate for picornaviruses. We reported that higher baseline expression of TLR3 was identified in patients with more rapid clearance of SVV-001, within the first week of administration; however, this expression did not appear to correlate with tumor response. Other potential biomarkers for SVV are the $\alpha 2,3$ - and $\alpha 2,6$-linked sialic acids, which have been reported to be necessary for SVV-001 infection in pediatric GBM cell lines, and the ratio of NEUROD1 to ASCL1 in SCLC, which may predict SVV-001 efficacy. ${ }^{13,19}$ If these data can be repeated across other tumors expressing neuroendocrine features and found to be predictive of infectivity, then they could be a possible biomarker used at the time of diagnosis or relapse.

As the supply of SVV-001 for clinical trials is limited and prior study results have not been promising enough, future studies in children may have to await greater clinical success with other oncolytic viruses. At least with regard to SVV-001, the patient's immune system appears to remain "King of the Hill", and until it can be dethroned via more effective immunomodulatory approaches to significantly blunt the rapidity of viral clearance and development of neutralizing antibodies, SVV-001 as an oncoviral therapy may have seen its last.

\section{Acknowledgment}

The Midwest Athletes Against Childhood Cancer (MACC) Fund supported this work.

\section{Disclosure}

The author reports no conflicts of interest in this work.

\section{References}

1. Hales LM, Knowles NJ, Reddy PS, Xu L, Hay C, Hallenbeck PL. Complete genome sequence analysis of Seneca Valley virus-001, a novel oncolytic picornavirus. J Gen Virol. 2008;89(pt 5):1265-1275.

2. Venkataraman S, Reddy SP, Loo J, Idamakanti N, Hallenbeck PL, Reddy VS. Structure of Seneca Valley virus-001: an oncolytic picornavirus representing a new genus. Structure. 2008;16(10):1555-1561.

3. Venkataraman S, Reddy SP, Loo J, Idamakanti N, Hallenbeck PL, Reddy VS. Crystallization and preliminary X-ray diffraction studies of Seneca Valley virus-001, a new member of the Picornaviridae family. Acta Crystallogr Sect F Struct Biol Cryst Commun. 2008;64(pt 4): 293-296.

4. Reddy PS, Burroughs KD, Hales LM, et al. Seneca Valley virus, a systemically deliverable oncolytic picornavirus, and the treatment of neuroendocrine cancers. $J$ Natl Cancer Inst. 2007;99(21):1623-1633.

5. Neotropix, Inc. Investigator Brochure NTX-010. Neotropix, Inc; 2010. Malvern, PA, USA.

6. Fitzgerald KD, Semler BL. Bridging IRES elements in mRNAs to the eukaryotic translation apparatus. Biochim Biophys Acta. 2009; 1789(9-10):518-528.

7. Belsham GJ. Divergent picornavirus IRES elements. Virus Res. 2009; 139(2):183-192.

8. Hellen CU, de Breyne S. A distinct group of hepacivirus/pestiviruslike internal ribosomal entry sites in members of diverse picornavirus genera: evidence for modular exchange of functional noncoding RNA elements by recombination. J Virol. 2007;81(11):5850-5863.

9. Willcocks MM, Locker N, Gomwalk Z, et al. Structural features of the Seneca Valley virus internal ribosome entry site (IRES) element: a picornavirus with a pestivirus-like IRES. J Virol. 2011;85(9):4452-4461.

10. Morton CL, Houghton PJ, Kolb EA, et al. Initial testing of the replication competent Seneca Valley virus (NTX-010) by the pediatric preclinical testing program. Pediatr Blood Cancer. 2010;55(2):295-303.

11. Yu L, Baxter PA, Zhao X, et al. A single intravenous injection of oncolytic picornavirus SVV-001 eliminates medulloblastomas in primary tumor-based orthotopic xenograft mouse models. Neuro Oncol. 2011;13(1):14-27. 
12. Poirier JT, Reddy PS, Idamakanti N, et al. Characterization of a fulllength infectious cDNA clone and a GFP reporter derivative of the oncolytic picornavirus SVV-001. J Gen Virol. 2012;93(pt 12):2606-2613.

13. Liu Z, Zhao X, Mao H, et al. Intravenous injection of oncolytic picornavirus SVV-001 prolongs animal survival in a panel of primary tumor-based orthotopic xenograft mouse models of pediatric glioma. Neuro Oncol. 2013;15(9):1173-1185.

14. Wadhwa L, Hurwitz MY, Chevez-Barrios P, Hurwitz RL. Treatment of invasive retinoblastoma in a murine model using an oncolytic picornavirus. Cancer Res. 2007;67(22):10653-10656.

15. Rudin CM, Poirier JT, Senzer NN, et al. Phase I clinical study of Seneca Valley virus (SVV-001), a replication-competent picornavirus, in advanced solid tumors with neuroendocrine features. Clin Cancer Res. 2011;17(4):888-895.
16. Burke MJ, Ahern C, Weigel BJ, et al. Phase I trial of Seneca Valley virus (NTX-010) in children with relapsed/refractory solid tumors: a report of the Children's Oncology Group. Pediatr Blood Cancer. 2015;62(5): 743-750.

17. Cerullo V, Diaconu I, Kangasniemi L, et al. Immunological effects of low-dose cyclophosphamide in cancer patients treated with oncolytic adenovirus. Mol Ther. 2011;19(9):1737-1746.

18. Miles LA, Brennen WN, Rudin CM, Poirier JT. Seneca Valley virus 3Cpro substrate optimization yields efficient substrates for use in peptide-prodrug therapy. PLoS One. 2015;10(6):e0129103.

19. PoirierJT,DobromilskayaI,MoriartyWF,PeacockCD,HannCL, RudinCM. Selective tropism of Seneca Valley virus for variant subtype small cell lung cancer. J Natl Cancer Inst. 2013;105(14):1059-1065.

\section{Publish your work in this journal}

Oncolytic Virotherapy is an international, peer-reviewed, open access online journal publishing original research, study protocols, reviews, editorials and commentaries on all aspects of oncolytic virology, namely the application of oncolytic viruses for the treatment of cancer. Specific topics in the journal include: Rationale and theoretical aspects of oncolytic virotherapy including in vitro, in vivo and mathematical

\section{Dovepress}

modeling; and practical application and problem solving in the clinic including identification of potential responders through biomarkers and genetic profiling. The manuscript management system is completely online and includes a very quick and fair peer-review system, which is all easy to use. Visit http://www.dovepress.com/ testimonials.php to read real quotes from published authors. 